\title{
Cross Section Prospects for MicroBooNE
}

\author{
Marco Del Tutto* \\ Department of Physics, University of Oxford, \\ Oxford OX1 3RH, United Kingdom \\ E-mail: marco.deltutto@physics.ox.ac.uk
}

MicroBooNE is a liquid-argon-based neutrino experiment, which began collecting data in Fermilab's Booster neutrino beam in October 2015. The physics goals of the experiment include probing the source of the anomalous excess of electron-like events in MiniBooNE. In addition to this, MicroBooNE is carrying out an extensive neutrino cross section physics program that will allow to investigate current theories on neutrino-nucleus interactions relevant to the neutrino oscillation program and in understanding the role played by nuclear effects in argon. These proceedings summarise the status of MicroBooNE's neutrino cross section analyses.

The 19th International Workshop on Neutrinos from Accelerators-NUFACT2017

25-30 September, 2017

Uppsala University, Uppsala, Sweden

\footnotetext{
* Speaker.

${ }^{\dagger}$ On behalf of MicroBooNE collaboration.
} 


\section{Introduction and Motivations}

The Micro Booster Neutrino Experiment (MicroBooNE) combines the physics goals of shortbaseline neutrino oscillations and neutrino cross section measurements with development goals to inform larger scale construction of Liquid Argon Time Projection Chambers (LArTPCs) for the long and short baseline neutrino program. MicroBooNE is located on-axis in the Booster Neutrino Beam (BNB) line at Fermilab, $470 \mathrm{~m}$ downstream from the target. MicroBooNE finished commissioning in summer 2015 and has been collecting neutrino data since October 2015 [1]. Many cross section analyses are currently underway within the MicroBooNE collaboration, including studies of charge-current $v_{\mu}$ interactions, proton multiplicity, charged-current $\pi^{0}$ production, neutral-current channels, and more. These proceedings describe the current status of the MicroBooNE cross section analyses.

Most of our foundational knowledge of neutrino cross sections in the $\sim 1 \mathrm{GeV}$ energy range comes from early experiments that collected relatively small data samples. These measurements were conducted in the 1970's and 1980's using either bubble or spark chamber detectors. These experiments provide the only single-nucleon experimental data currently available [2].

Modern experiments use heavy nuclei (usually hydrocarbon, water or argon) as target materials. In addition to scattering off individual nucleons in the nucleus, it has been recently recognised that neutrinos can also scatter off of nucleons belonging to correlated pairs (short-range nucleonnucleon correlations) and to two-nucleon currents arising from meson exchange (MEC). This leads to multi-nucleon or $n p-n h$ excitations, [2,3].

In addition to having measured such nuclear effects, modern experiments have also reported single and double differential cross sections for the first time with high statistics. The most recent experiments (MiniBooNE, MINERvA, T2K) have difficulties to provide definitive answers to the modelling issues for quasi-elastic reactions. The MiniBooNE experiment [4] measured for the first time double differential cross section as a function of muon angle and kinetic energy for muon neutrino charged-current without pions $(\mathrm{CC} 0 \pi)$ interactions. The MINER $v$ A experiment $[5,3]$ has measured double differential cross section as a function of transverse $p_{T}$ and longitudinal $p_{L}$ muon momentum, and the T2K experiment [6] measured double differential cross sections as a function of muon momentum and angle. Although there is still tension between several models, for all these experiments it is evident that nuclear effects have to be included to reach agreement with the measurements.

The ArgoNeuT experiment (a LArTPC experiment in the Fermilab NuMI beam) performed a proton multiplicity measurement [7], and has measured the $\mathrm{CC} 0 \pi$ cross section for both neutrino and anti-neutrino data. They found that GENIE predictions are 64\% and 22\% higher than data for neutrinos and anti-neutrinos respectively. These results hint that more accurate final state interaction (FSI) models are needed in the generators and additional experimental data on argon is needed to cross-check these results at other energies and constrain nuclear models. ArgoNeuT was able to detect and study low energy protons opening up many possibilities for studying nuclear effects. ArgoNeuT, however, had very low statistics. With a much larger detector and more beam exposure, MicroBooNE collects significantly higher statistics data, and will be able to study these effects in greater detail. 


\section{The MicroBooNE Experiment}

MicroBooNE is the first large (89 tons of active mass) LArTPC to operate in the United States, with dimensions of 10.4 (length) $\times 2.5$ (width) $\times 2.3$ (height) $\mathrm{m}$. In the MicroBooNE LArTPC detector [1] charged particles traversing a volume of highly-purified liquid argon leave trails of ionisation electrons in their wake and also create prompt vacuum ultraviolet scintillation photons. The ionisation trails are transported over distances of the order of meters under the influence of a uniform electric field until they reach anode planes located along one side of the active volume. The anode planes are composed of wires that receive the signals induced by the ionisation electrons drifting towards them. The charged particle trajectory reconstruction is derived from the known wire positions within the anode planes and the drift time of the ionisation. The drift time is the difference between the arrival times of ionisation signals on the wires and the $t_{0}$ time the interaction took place in the detector which is established by a trigger provided by the light collection system: 32 photo-multipliers (PMTs) located on one side of the TPC volume.

MicroBooNE is a surface detector and is therefore subject to constant bombardment by cosmic radiation (or cosmics) from the atmosphere. Quantitatively, this amounts to $\sim 5 \mathrm{kHz}$, or $\sim 20-30$ cosmics per MicroBooNE's $4.8 \mathrm{~ms}$ readout time window. However, neutrinos are not delivered continuously but in distinct spills. The BNB spill $(1.6 \mu \mathrm{s})$ is much smaller than the drift time, and a natural way to separate cosmics from neutrino interactions is to compare the time of the activity in the detector with the spill trigger time window. The light collection system signals are then vital in distinguishing detector activity that is in-time with the beam from that which is out-of-time. The scintillation light is fast compared to the ionisation electrons. Spatial information can also be inferred from the light collection signals, further aiding in the eventual reconstruction of the activity occurring inside the detector.

\subsection{Physics Goal}

One of the main physics goals of MicroBooNE is to perform high-statistics precision measurements of $v$-Ar interactions in the $1 \mathrm{GeV}$ range. Cross section measurements allow us to better understand neutrino interaction models and nuclear effect which are of fundamental importance for oscillation experiments.

MicroBooNE, due to its large size and excellent performance of the Fermilab accelerator complex, is collecting large statistics and most analyses are expected to eventually be systematics limited. MicroBooNE will collect around 170,000 $v_{\mu}$ CC inclusive interactions in 3 years, opening a new opportunity to study nuclear effects in argon. MicroBooNE is able to detect both neutralcurrent (NC) and charged-current (CC) events. Figure 1 shows two candidate neutrino events in MicroBooNE: a CC-multitrack (left) and a CC- $1 \pi$ (right) event.

There are several ways to test nuclear models, and the MicroBooNE cross section program has currently several on-going analyses:

- $v_{\mu}$ CC inclusive double differential cross section: this will provide a large sample of neutrinoinduced muons, and so it will allow a detailed study of the kinematic properties of final state muons (much like the MiniBooNE, T2K, and MINER $v$ A measurements, except on argon). It is a good channel to compare to other experiments and theories because it has such a clearly 

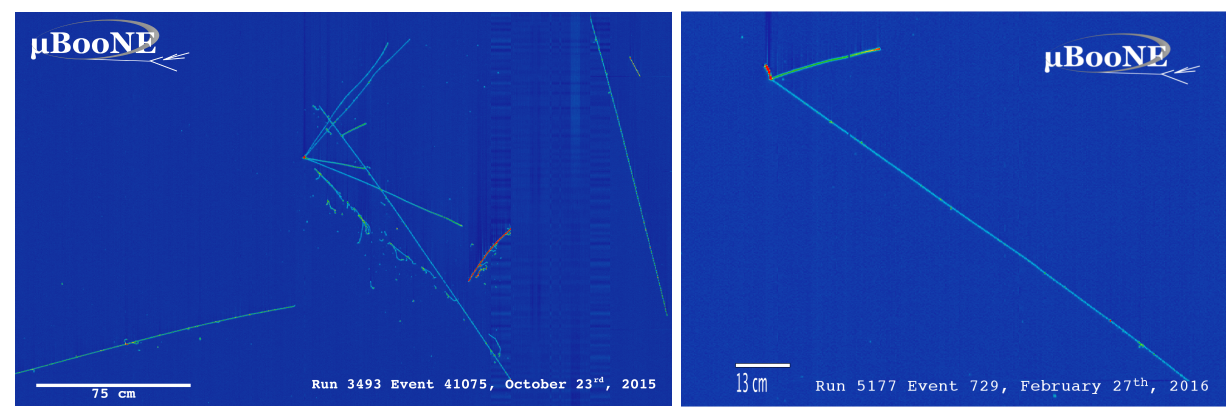

Figure 1: Event displays showing neutrino interactions in MicroBooNE. Left is a CC-multitrack and right a CC- $1 \pi$ candidate event. These are collection plane images. Neutrino beam is coming from the left. $x$ axis shows the collection plane wires, $y$ axis shows the drift time. Colour indicates amount of deposited charge on the wires.

defined (inclusive) signature. It will also provide a well defined $v_{\mu} \mathrm{CC}$ sample for other exclusive analyses.

- $v_{\mu}-C C 1 p$ and $v_{\mu}-C C 2 p$ : these analysis are a subset of the previous channel. Different nuclear effects can be studied by looking at the number of particles in the final state, this is why these analyses focus on selecting a determined number of protons in the final state.

- $v_{\mu}$ CC final state particle multiplicity: this analysis starts from a CC inclusive event selection and its goal is to measure the number of track-like final state particles (lepton included).

- $v_{\mu}$-CC $\pi^{0}$ : looks for events with a muon track and two showers originating from a common vertex. It will provide a vital photon sample for electron/gamma separation studies and a sample of $\pi^{0}$ s to study reconstruction efficiencies for $\mathrm{NC} \pi^{0}$ interactions, expected to be a background to $v_{e}$ appearance signal.

- NC elastic interactions: proton identification is crucial for this analysis, and an improved proton identification developed for this analysis can also benefit the aforementioned CC analyses.

\section{Muon Neutrino Charged-Current Inclusive Analysis}

In order to perform high-statistics physics measurements, MicroBooNE requires automated selection algorithms, which in turn require automated reconstruction as input. MicroBooNE has developed two complementary (preliminary) selections to select charged-current muon neutrino interactions in the liquid argon. Both are fully automated, cut-based selections. The results presented here are those given by the second selection, but the full details of both selections can be found in a MicroBooNE public note [8]. More details on this event selection and performances can be found in reference [9].

Applying this selection to a subset of data equivalent to $5 \times 10^{19}$ protons on target, and performing a background subtraction of the surviving cosmics outside the beam window using beamoff data, we observe the kinematic distribution of the muon momentum shown in Figure 2. These distributions are presented with statistical uncertainties only, and with Monte Carlo distributions 
scaled to the same number of events as the data; the assessment of systematic uncertainties is still ongoing. The on-beam data sample contains events where no neutrino interacted in the detector (a purely cosmic event) and events where a neutrino interaction is also present. Off-beam data are taken outside of the beam spill window and contain only cosmic events. The subtraction of off-beam from on-beam data subtracts the background of events where no neutrino interaction is present. In the remaining events a neutrino interaction is present, but a cosmic in the same event may still be selected, hence the cosmic background in figure 2. A third and final CC inclusive selection, which combines and improves upon these two selections, is currently underway.

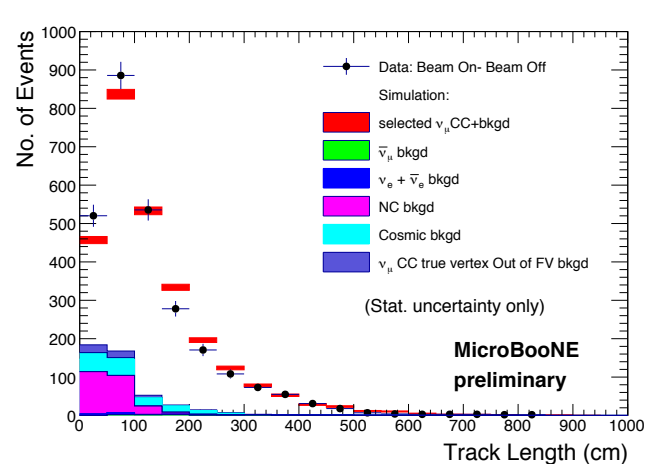

Figure 2: The black data points symbolise on-beam minus off-beam data with statistical error bars. The red shaded histogram represents the Monte Carlo prediction, with the bands representing the statistical uncertainty only. The backgrounds contained in the red are additionally shown in different colours corresponding to different physics processes. Backgrounds are stacked. The simulation is normalised to the same number of total events as the data.

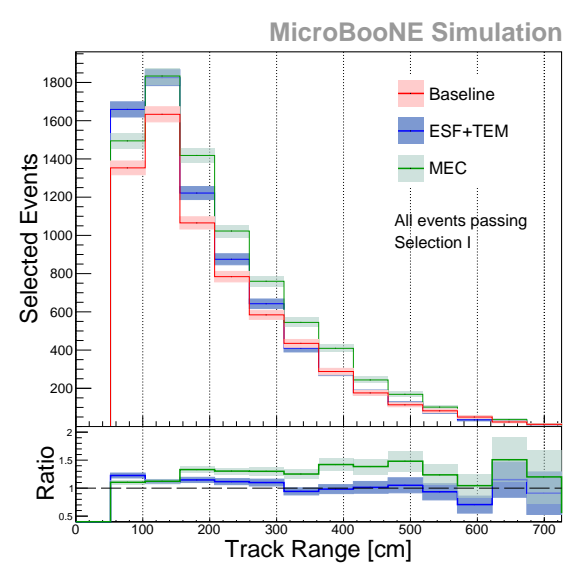

Figure 3: Simulated muon track length distribution with different nuclear models in the neutrino simulation. The green distribution shows the effect of simulating MEC interactions in addition to the nominal GENIE model. The blue shows the effect of using the ESF+TEM model. Uncertainties are statistical only.

At high neutrino energies, a Relativistic Fermi Gas (RFG) model of the nucleus, and a dipole axial form factor with axial mass of approximately $1 \mathrm{GeV}$, was found to agree well with data collected by previous experiments using lighter target nuclei. In observations of CCQE events at MiniBooNE and other experiments, this model was found to not agree well with the data using heavier nuclear targets, and fits resulted in an effective axial mass value of $1.35 \mathrm{GeV}$. For this and the following in this section, see [10] and references therein.

Rather than introducing an effective parameter, additional processes were proposed proposed which would address the discrepancies seen in the MiniBooNE data. MEC is one way to model an additional process, where a neutrino interacts with a correlated pair of nucleons (which may be in a quasi-deuteron state). Figure 3 shows the baseline simulation in red and with MEC interactions in green. The effect is an increase on the total number of expected selected events of $24 \%$. The Transverse Enhancement Model (TEM) is another way to model the nuclear effects. In this model, the transverse cross section is enhanced based on observations in electron scattering data. We combined TEM with the effective spectral function (ESF), which replaces the Relativistic Fermi Gas model used in all other samples as the GENIE default. Figure 3 shows the simulation with ESF and TEM in blue. The effect is an increase on the total number of expected selected events of 


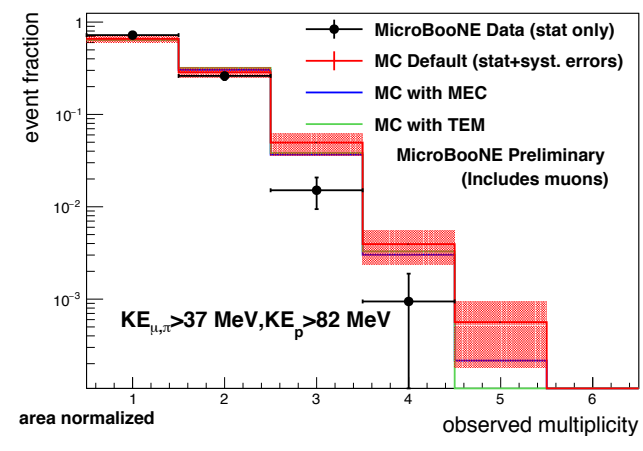

Figure 4: Observed neutrino multiplicity distributions for MicroBooNE data overlaid with three GENIE predictions. The distributions are area normalised and cosmicy-ray-background subtracted. Data error bars include statistical uncertainties only. Monte Carlo error bands include MC statistical uncertainties and systematic uncertainty contributions added in quadrature [11].

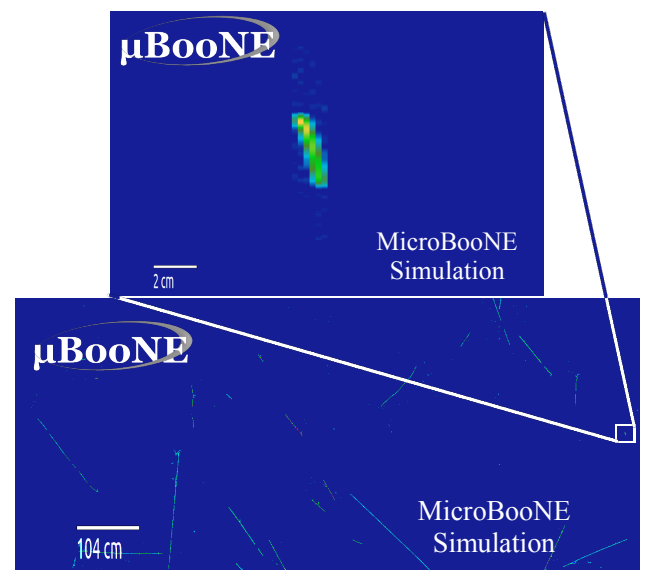

Figure 5: 2D event display of a simulated neutralcurrent elastic event in MicroBooNE that was classified as a proton. The top image is a close-up event display of the simulated proton track. The bottom image shows the side view of the entire MicroBooNE TPC. All of the additional tracks are from cosmic rays [12].

$13 \%$. MicroBooNE will be able to distinguish these models significantly as we analyse additional data, and move to absolutely normalised data and MC distributions.

\section{Charged Particle Multiplicity Analysis}

The charged particle multiplicity, or number of primary charged particles emerging from neutrino interactions, is a simple observable characterising final states in collision processes, including neutrino interactions. In MicroBooNE, the observable charged particle multiplicity measures the number of reconstructed charged particles exiting the target nucleus participating in the neutrino interaction. This analysis [11] starts with the $v_{\mu}$ CC event selection, described in the previous section, in order to select $v_{\mu}$ candidates. A set of conditions are then applied to ensure a high quality muon track candidate and a "muon directionality classifier" is implemented that categorises the final selected events into four sub-samples for the purpose of cosmic-ray background estimation.

The area normalised multiplicity distributions from three different GENIE predictions overlaid on data is shown in Figure 4. The kinetic energy threshold ranges from $37 \mathrm{MeV}$ for a pion to 82 $\mathrm{MeV}$ for a proton. The distributions have not been corrected for non flat acceptance and efficiency effects. Is is found that all three GENIE tunes are consistent within uncertainties with the data. The two alternative GENIE models considered here are not expected to yield significant multiplicity distribution differences; they were chosen to be used in this analysis simply because of their availability, and this analysis serves as proof of principle for future comparisons involving more model predictions. MicroBooNE will improve this analysis in the future by including larger statistics, improving particles identification and by reducing thresholds on the particles kinetic energies. This is the first time that such a multiplicity distribution has been measured in neutrino-argon interactions. 


\section{Proton and Neutral-Current Identification}

Measuring particle multiplicities implies being able to also reconstruct short particles tracks coming out of the interaction vertex. Low energy protons usually produce very short tracks and are difficult to identify. Figure 5 shows the full MicroBooNE read-out window, while the inset shows a simulated proton from a neutral-current elastic neutrino interaction. Neutral-current elastic interactions are the most difficult to detect automatically because there is only one visible particle coming from the interaction vertex. There is no unique topology separating these events from the cosmic background. The current reconstruction efficiency for tracks from NC elastic proton events in simulation is approximately 50\%, and this number is rapidly improving. Once tracks are reconstructed we attempt to identify the type of particle and interaction that produced them. In the $\mathrm{NC}$ elastic case, we want to specifically select proton tracks. To identify proton tracks, we currently use a gradient-boosted decision tree classifier [12]. The classifier takes reconstructed tracks as input and uses features such as track position, angle, shape, charge and others to classify tracks as protons. Of the reconstructed test tracks that were input to the classifier, $84 \%$ of the protons from simulated neutrino interactions, and $63 \%$ of the protons from simulated cosmic interactions were classified correctly as protons. Of the reconstructed test tracks that were classified as protons, $89 \%$ were true simulated protons ( $22 \%$ neutrino induced protons and $67 \%$ cosmic induced protons).

\section{Conclusion}

These proceedings described several cross section analyses ongoing at MicroBooNE, which has currently measured muon-neutrino kinematic distributions and charged particle multiplicity. These measurements demonstrate that a large-scale liquid argon TPC can make excellent measurements of neutrino interactions, and provide the foundation for a much more wide-ranging programme of cross section measurements to come.

\section{References}

[1] MicroBooNE Collaboration, JINST 12, P02017, 2017

[2] J.A. Formaggio, G.P. Zeller, 2013, Rev. Mod. Phys. 84, 1307, 2012

[3] L. Alvarez-Ruso et al., NuSTEC White Paper, 2017, https : / / arxiv . org/abs / 1706.03621

[4] A.A. Aguilar-Arevalo et al. (MiniBooNE Collaboration), Phys. Rev. D 81, 0920052010

[5] M. Betancourt, JPS Conf. Proc. 12, 010016, 2016

[6] K. Abe et al. (T2K), Phys. Rev. D 93, 112012, 2016

[7] O. Palamara, JPS Conf. Proc. 12, 010017, 2016

[8] MicroBooNE Collab., Public Note 1010, microboone.fnal.gov/public-notes/

[9] M. Del Tutto, 52 ${ }^{\text {nd }}$ Recon. de Moriond 2017, https: / / arxiv. org/abs/1705. 04894

[10] M. Del Tutto et al., Journal of Physics: Conference Series 888, 012140, 2017

[11] MicroBooNE Collab., Public Note 1024, microboone. fnal.gov/public-notes/

[12] MicroBooNE Collab., Public Note 1025, microboone. fnal.gov/public-notes / 